\title{
Smoking before the birth of a first child is not associated with increased risk of breast cancer: findings from the British Women's Heart and Health Cohort Study and a meta-analysis
}

\author{
DA Lawlor",', S Ebrahim' and G Davey Smith' \\ 'Department of Social Medicine, University of Bristol, Canynge Hall, Whiteladies Rd, Bristol BS7 8QA, UK
}

It has been suggested that the period between puberty and first birth is a time when the breast is particularly susceptible to carcinogenic effects. In a cohort of 3047 women aged 60-79 years ( $N=139$ breast cancer cases), we found no association between smoking before the birth of a first child and breast cancer risk: fully adjusted (for age, number of children, age at birth of first child, age at menarche, age at menopausal, hysterectomy and/or oophorectomy, ever use of oral contraception, use of hormone replacement therapy, alcohol consumption, body mass index, childhood and adulthood social class) odds ratio 1.06 (95\% confidence interval: 0.72 , I.56). The pooled estimate from a meta-analysis of our study and II previously published studies $(N=6528$ cases $)$ was I.07 (0.94, 1.22). We conclude that smoking prior to the birth of a first child is not associated with increased risk of breast cancer.

British Journal of Cancer (2004) 91, 512-518. doi:10.1038/sj.bjc.6601916 www.bjcancer.com

Published online 29 June 2004

(c) 2004 Cancer Research UK

Keywords: smoking; breast cancer; first pregnancy; epidemiology

It has been suggested that smoking may play differing roles in the aetiology of breast cancer depending upon the period in a woman's life during which she smokes (Terry and Rohan, 2002). Breast tissue may be most susceptible to environmental carcinogens during rapid cell proliferation and before complete cellular differentiation, that is, between puberty and completion of a woman's first pregnancy. This suggestion is supported by the fact that older age at menarche and early age at first pregnancy, both of which will decrease the length of this susceptible period, are associated with reduced risk of breast cancer (MacMahon et al, 1970; Hsieh et al, 1990).

A small number of studies that have assessed the association of smoking prior to completion of first pregnancy with breast cancer and have reported conflicting results (Terry and Rohan, 2002). We aimed to assess this association in a large cohort study and in a meta-analysis of all previously published studies.

\section{MATERIALS AND METHODS}

\section{British Women's Heart and Health Cohort Study}

Data from the baseline assessment and first 3 years of follow-up of the British Women's Heart and Health study were used. Full details of baseline assessment have been reported previously (Lawlor et al, 2003a,b). In brief, 4286 women were assessed (medical record review, research nurse interview, self-completed questionnaire and physical examination) between 1999 and 2001. These women have

*Correspondence: Dr DA Lawlor; E-mail: d.a.lawlor@bristol.ac.uk Received 3 February 2004; revised 19 April 2004; accepted 21 April 2004; published online 29 June 2004 been followed up over a median of 3.5 years by flagging with the NHS central register for mortality and cancer register data, two yearly review of their primary-care medical records (in Britain primary-care records contain details of secondary-care treatment) and a recently mailed 3-year follow-up health questionnaire (Q3) sent to all surviving participants between March and September 2003. Of the 4108 survivors, $3704 \quad(90 \%)$ responded to this questionnaire. Ethics committee approval was obtained for this study.

\section{Breast cancer cases}

In this paper, we have included all cases of breast cancer reported at baseline assessment (prevalent cases) and those occurring over the median 3.5 years follow-up period (incident cases). Three main sources were used to determine breast cancer status: (i) self-report in the baseline questionnaire and Q3; (ii) a diagnosis recorded in the medical records and (iii) all participants were flagged with the National Health Service central register (NHSCR) that provided details of cancer registrations. Anyone with a diagnosis of breast cancer from any one of these three sources was considered to be a case. Five additional incident cases that had not been identified by any of these three sources were obtained because they occurred as the underlying cause or elsewhere on the woman's death certificate. Thus, this study is of prevalent and incident breast cancer cases occurring any time in the woman's life up to January 2004.

\section{Assessment of smoking and potential covariates}

Age at menopause, age at menarche, number of pregnancies and live births, current and past use of hormone replacement therapy (HRT) and past use of oral contraception, history of a 
hysterectomy and/or oophorectomy, alcohol and smoking history were obtained from the self-completed questionnaire and/or the research nurse interview (Lawlor et al, 2003a, b). Detailed smoking histories included the age at which smoking started. Adult social class was defined on the basis of the longest held occupation of her husband for married women and her own longest held occupation for single women, childhood social class was defined on the basis of the longest held occupation of the participants father, and both were classified according to the Registrar General's classification (Office of Population Censuses and Surveys, 1980). Standing height was measured without shoes using a Harpenden Stadiometer, which recorded to the nearest millimetre. Weight was measured in light clothing without shoes to the nearest $0.1 \mathrm{~kg}$ using Soenhle portable scales.

\section{Statistical analysis}

Multiple logistic regression was used to assess the association between smoking in relation to first birth and breast cancer with adjustment for potential confounding factors: age (entered as a continuous variable), age at first birth (indicator: $<20,21-24,25-$ $29,30-34, \geqslant 35$ ), age at menarche (indicator: $<11,11,12,13,14$, $\geqslant 15$ ), number of births (indicator: $1,2,3,4,5,6+$ ), age at menopause (indicator: $<40,41-44,45-49,50,51$, older than 51), use of HRT (ever or never), hysterectomy and/or oophotectomy (binary), childhood and adult social class (indicator: I, II, IIInonmanual, IIImanual, IV, V, unemployed), body mass index (BMI) (continuous) and alcohol consumption (indicator: daily, weekends only, once or twice a month, special occasions, never). To further explore whether smoking around the time of breast development was a risk factor for future breast cancer, we assessed the association of smoking during the period 1 year before menarche and the first 5 years after menarche in all women using multiple logistic regression. For all analyses robust standard errors, which take into account possible nonindependence between women from the same town, were used to calculate confidence intervals (CI) and $P$-values.

\section{Meta-analysis}

Searches of Medline and Embase (up to January 2004) were undertaken using extended terms for breast neoplasia and smoking. Any published study that assessed the association between smoking before a first pregnancy and breast cancer risk was included in a meta-analysis. We decided a priori to pool estimates using DerSimonian and Laird's random effects methods, since it was likely that there would be heterogeneity between studies due to differences in the number of pre- and postmenopausal cases and differences in study design (DerSimonian and Laird, 1986). Metaregression analysis was used to assess the effect of menopausal status on heterogeneity between studies (Sterne et al, 2001). A series of sensitivity analyses were undertaken in which: (i) two studies with in-pregnancy smoking assessment only were excluded; (ii) one study with smoking only before pregnancy was excluded; (iii) for studies that provided estimates of both any smoking before pregnancy and only smoking before pregnancy, the latter estimates were substituted into the main analysis; (iv) for studies with varying durations of smoking before pregnancy these were substituted into the main analysis starting first with shortest duration. We examined funnel plots and used Egger and Begg tests to determine the extent of small study bias. (Sterne et al, 2001) All statistical analyses were undertaken using Stata version 8 .

\section{RESULTS}

Of the 4286 women in this study, 225 (5.3\%) had breast cancer ascertained from at least one source. The majority $(82 \%)$ of these cases were identified from at least two sources. The age distribution of women with cancer identified by each source were similar - mean (standard deviation) age of women with breast cancer identified by self report 68.7 (5.3), identified by medical record review 68.9 (5.3) and identified by cancer register 69.0 (5.4). Of the 225 cases, 170 were prevalent and 55 incident cases.

Table 1 shows the distributions of established breast cancer risk factors and other potential risk factors by breast cancer status among all 4286 women. For established risk factors, our results are in the same direction and of similar magnitudes to what one would expect based on consistent results from previous studies. None of the smoking alcohol or social class variables were associated with breast cancer.

\section{Smoking prior to first birth}

Of the 4286 women, 3467 (81\%) had at least one live birth and of these $3047(88 \%)$ gave their age at the time that their first child was born. The associations presented in Table 1 were similar in this subgroup of 3047 women. Of these 3047 women, 139 (4.6\%) had breast cancer. Table 2 shows breast cancer occurrence and the distributions of potential confounding factors by smoking in

Table I Smoking and other characteristics irrespective of whether women had breast cancer or not $(N=4286)$

\begin{tabular}{|c|c|c|c|}
\hline & \multicolumn{2}{|c|}{ Percent (95\% CI) } & \multirow[b]{2}{*}{$P$-value } \\
\hline & Breast cancer $N=225$ & No breast cancer $N=4061$ & \\
\hline Ever smoked & $43.1(36.5,49.9)$ & $44.8(43.2,46.3)$ & 0.63 \\
\hline Current smoker (at baseline assessment) & $1 \mid .6(7.7,16.5)$ & $12.0(11.0,13.1)$ & 0.84 \\
\hline Smoked prior to first birth ${ }^{\mathrm{a}}$ & $32.1(24.5,40.6)$ & $30.5(28.9,32.2)$ & 0.68 \\
\hline Smoked either I year before or within 5 years after menarche ${ }^{b}$ & $25.6(19.2,32.8)$ & $26.3(24.7,27.9)$ & 0.84 \\
\hline Nulliparous & $13.8(9.4,19.3)$ & $9.5(8.6,10.5)$ & 0.04 \\
\hline Young age and menarche ( $<||$ years) & $4.9(2.5 .8 .6)$ & $2.1(1.7,2.6)$ & 0.008 \\
\hline Old age at menopause ( $>51$ years) & $33.8(27.6,40.04)$ & $27.2(25.9,28.6)$ & 0.03 \\
\hline Hysterectomy and/or oophorectomy & $24.4(19.0,30.6)$ & $30.2(28.8,31.6)$ & 0.06 \\
\hline Ever used HRT & $18.7(13.8,24.4)$ & $12.5(\mid 1.5,13.6)$ & 0.007 \\
\hline Ever used oral contraception & $25.4(19.7,31.7)$ & $23.9(22.2,24.9)$ & 0.53 \\
\hline Childhood manual social class & $81.3(75.6,86.2)$ & $80.0(78.7,81.1)$ & 0.61 \\
\hline Adult manual social class & $55.1(48.4,61.7)$ & $57.4(55.9,58.9)$ & 0.49 \\
\hline Over weight $(\mathrm{BMI}>25)$ & $71.4(64.8,77.1)$ & $67.9(66.4,69.4)$ & 0.30 \\
\hline Daily alcohol consumption & $21.0(15.8,27.1)$ & $18.1(16.9,19.4)$ & 0.29 \\
\hline Tee-total & $17.8(12.9,23.5)$ & $16.5(15.3,17.7)$ & 0.63 \\
\hline
\end{tabular}

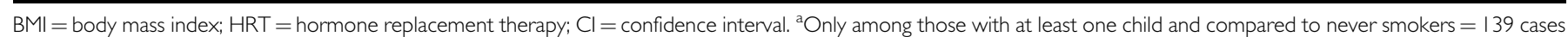
(see text). 'Irrespective of whether they had children or not and compared to never smokers. 
relation to first birth. There was no association between breast cancer and smoking in relation to first birth. The age-adjusted odds ratio for smoking before the first birth was 1.04 (95\% CI: 0.71, 1.51 ) and the fully adjusted (age, number of children, menarcheal and menopausal age, hysterectomy, use of hormone replacement, use of the oral contraceptive pill, childhood and adulthood social class and BMI) odds ratio was $1.06(0.72,1.56)$. When the analyses were restricted to those who only smoked before their first pregnancy (ie excluding from the analyses all women who smoked but only began after their first birth), the association was essentially unchanged: fully adjusted odds ratio $1.04(0.67,1.59)$. The age-adjusted odds ratios for women who smoked, but only after the birth of their first child compared to never smokers, was $0.79(0.44,1.41)$ and the fully adjusted odds ratio for this association was $0.70(0.36,1.36)$.

\section{Smoking around the time of puberty}

Among all women in the cohort (4286 women with 225 cases of breast cancer), the age-adjusted odds ratio for smoking either in the year prior to or within 5 years of menarche compared to never smoking was 0.96 . $(0.69,1.36)$; the fully adjusted odds ratio was $1.00(0.70,1.39)$.

\section{Sensitivity analyses}

In the whole cohort, $186(83 \%)$ of the total 225 cases were postmenopausal and in the subgroup of women with at least one birth and a known age at first birth 118 (85\%) of the 139 breast cancers were postmenopausal. We were therefore unable to determine with any level of precision the effect of associations on premenopausal cancers. The associations for postmenopausal breast cancers only did not differ from those presented and the point estimates for premenopausal cancers were similar to those for postmenopausal cancers and close to the null value (point estimate for premenopausal cancers associated with smoking prior to first birth $=0.96$, and for smoking around the time of puberty $=0.94)$. When the analyses were restricted to incident cases only, although considerably less precise, the results did not differ from those presented: fully adjusted odds ratio $1.08(0.39$,

Table 2 Breast cancer and other characteristics by smoking in relation to birth of first child among women with at least one live birth and who provided age at first birth $(N=3047)$

\section{Percent $(95 \% \mathrm{Cl})$}

Never smoked $N=1739$ Smoked before first birth $N=933$ Smoked but only after first birth $N=376 P$-value

\begin{tabular}{|c|c|c|c|c|}
\hline Breast cancer & $4.7(3.8,5.8)$ & $4.8(3.6,6.4)$ & $3.7(2.2,6.2)$ & 0.66 \\
\hline Young age and menarche ( $<||$ years) & $2.2(1.6,3.0)$ & $2.1(1.4,3.3)$ & $2.1(1.1,4.2)$ & 0.99 \\
\hline Hysterectomy and/or oophorectomy & $30.9(28.8,33.2)$ & $29.3(26.4,32.3)$ & $34.6(29.9,39.5)$ & 0.17 \\
\hline Ever used HRT & $13.4(11.9,15.1)$ & $16.6(14.4,19.1)$ & $12.8(9.8,16.5)$ & 0.05 \\
\hline Childhood manual social class & $78.9(76.9,80.8)$ & $79.4(76.7,81.9)$ & $84.3(80.3,87.6)$ & 0.05 \\
\hline Adult manual social class & $55.4(53.0,57.7)$ & $55.3(52.1,58.5)$ & $65.4(60.5,70.1)$ & 0.001 \\
\hline Over weight $(\mathrm{BMI} \geqslant 25)$ & $69.7(67.4,71.8)$ & $66.2(63.0,69.3)$ & $73.9(68.9,78.3)$ & 0.03 \\
\hline
\end{tabular}

$\mathrm{BMI}=$ body mass index; HRT = hormone replacement therapy; $\mathrm{Cl}=$ confidence interval.

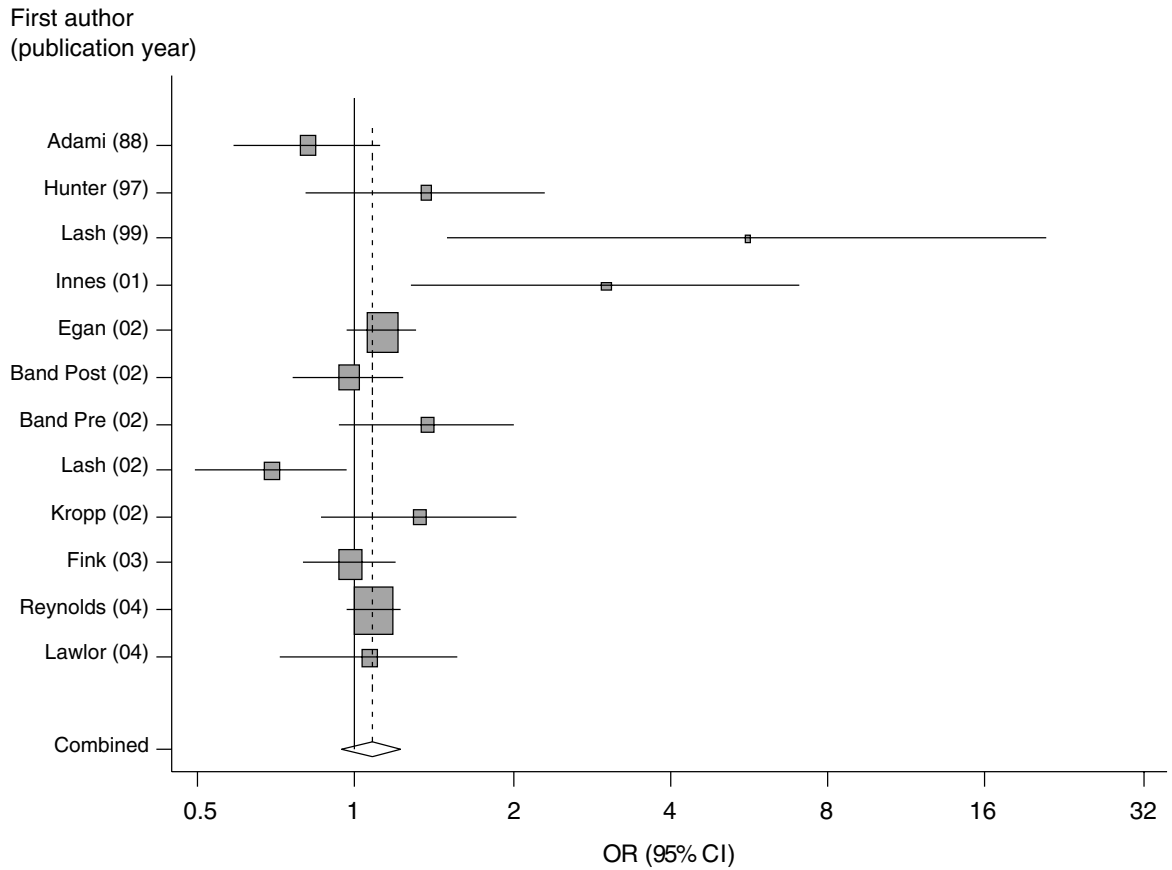

Figure I Meta-analysis of studies assessing the effect of smoking before/during first pregnancy with breast cancer risk. 
Table 3 Summary of studies assessing the association of smoking prior to the birth of a first baby and breast cancer risk

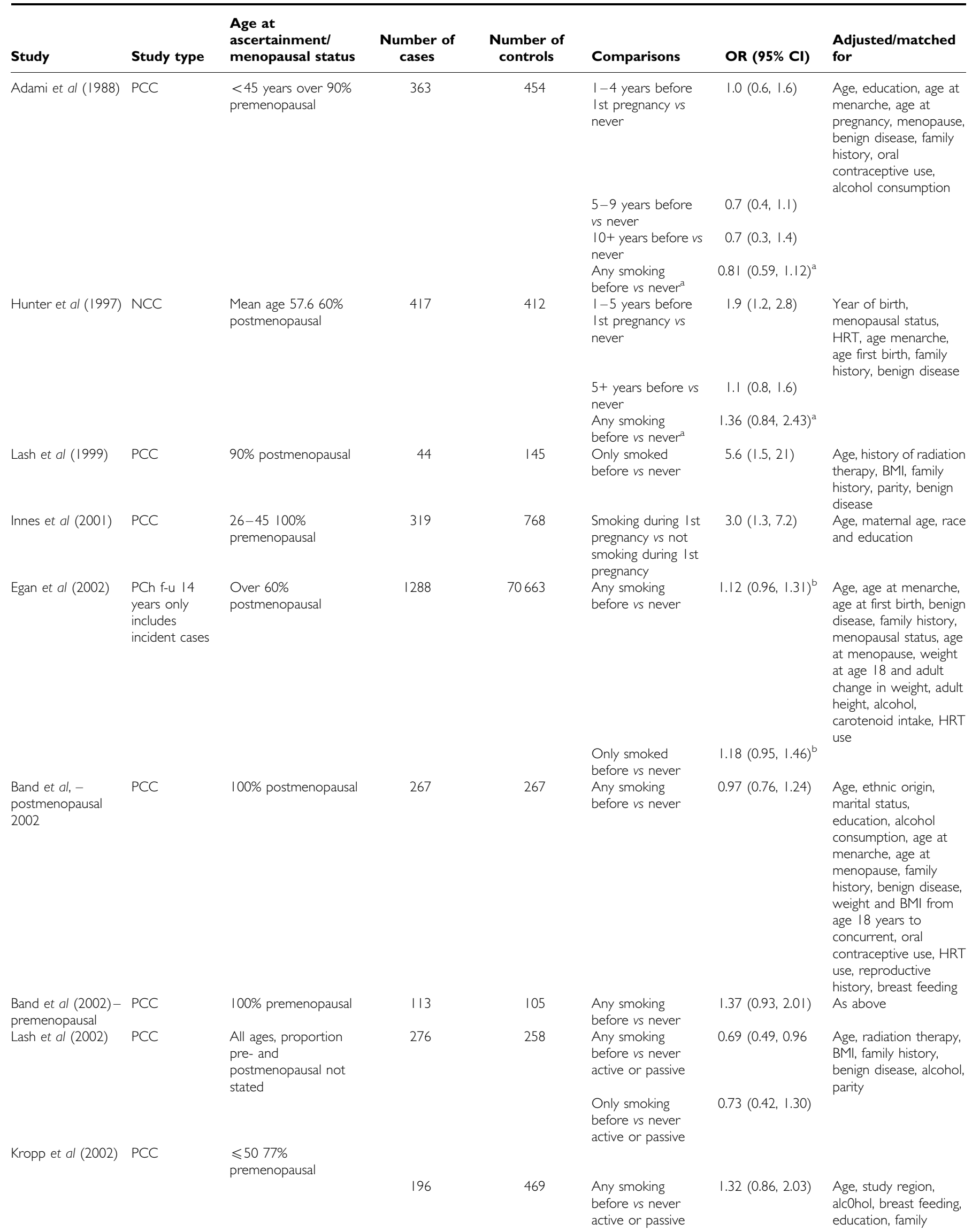


Table 3 (Continued)

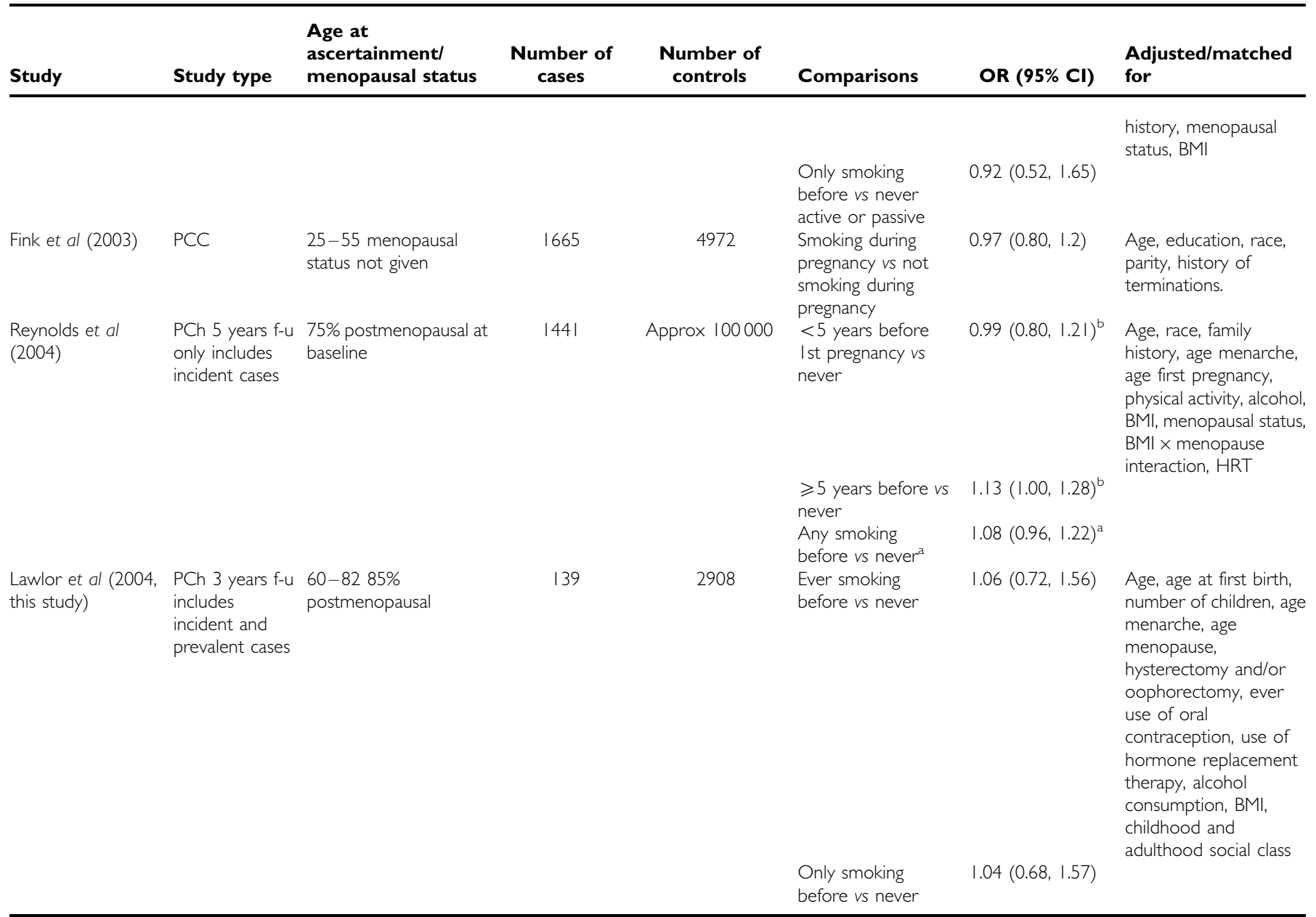

OR: odds ratio; Cl: confidence interval; NCC: nested case-control - this study was nested in a population, prospective cohort; PCC: population-based case-control; PCh: population cohort; HRT: hormone replacement therapy, BMI: body mass index; f-u: follow-up. ${ }^{a}$ Not provided in the paper but calculated from the data provided - these values used in meta-analysis. ${ }^{b}$ Hazard ratios.

2.55). When all analyses were repeated using breast cancer data from each of just one of the three sources (self-report, medical records, cancer register), the results were unchanged.

\section{Meta-analysis}

We identified 11 studies in 10 publications; these are summarised in Table 3. (Adami et al, 1988; Hunter et al, 1997; Lash and Aschengrau, 1999; Innes and Byers, 2001; Egan et al, 2002; Band et al, 2002; Lash and Aschengrau, 2002; Kropp and Chang-Claude, 2002; Fink and Lash, 2003; Reynolds et al, 2004) The pooled analysis for these 11 studies, together with the study presented here, included 6528 breast cancer cases and provided an odds ratio $(95 \% \mathrm{CI})$ of $1.07(0.94,1.22)$ (Figure 1$)$. The pooled estimates from differing sensitivity analyses to assess the effect of different exposure measures did not differ substantively from this estimate (all odds ratios for these analyses were between 1.05 and 1.08). There was heterogeneity between the studies $(P=0.01)$, which was not explained by menopausal status $(P=0.34)$. The Egger test did not suggest strong evidence of small study bias $(P=0.23)$, although the Begg test provided more evidence of this $(P=0.07)$. The funnel plot (Figure 2) shows the influence of two small studies with large positive effects on the Begg test result, and examination of the Forrest plot (Figure 1) suggests that these two studies are an important source of heterogeneity. When these two studies were removed from the meta-analysis (leaving $N=6165$ cases), the pooled odds ratio $(95 \% \mathrm{CI})$ was $1.03(0.93,1.14)$ with no strong evidence of heterogeneity between studies $(P=0.23)$. Other than being two of the smallest and least precise studies, it can be seen from Table 3 that there is nothing that makes these two studies specifically different from all other studies.

\section{DISCUSSION}

Our results suggest that there is no association between a woman smoking before the birth of her first child and breast cancer. Our finding that smoking around the time of puberty was not associated with increased risk of breast cancer is consistent with a number of other studies.(O'Connell et al, 1987; Rohan and Baron, 1989; Chu et al, 1990; Ewertz, 1990, 1993; Field et al, 1992; Smith et al, 1994; Baron et al, 1996; Band et al, 2002) Taken together, these findings suggest that smoking during the period of breast tissue development and before final cellular differentiation (ie before completion of a first pregnancy) is not associated with breast cancer. 
Begg's funnel plot with pseudo-95\% confidence limits

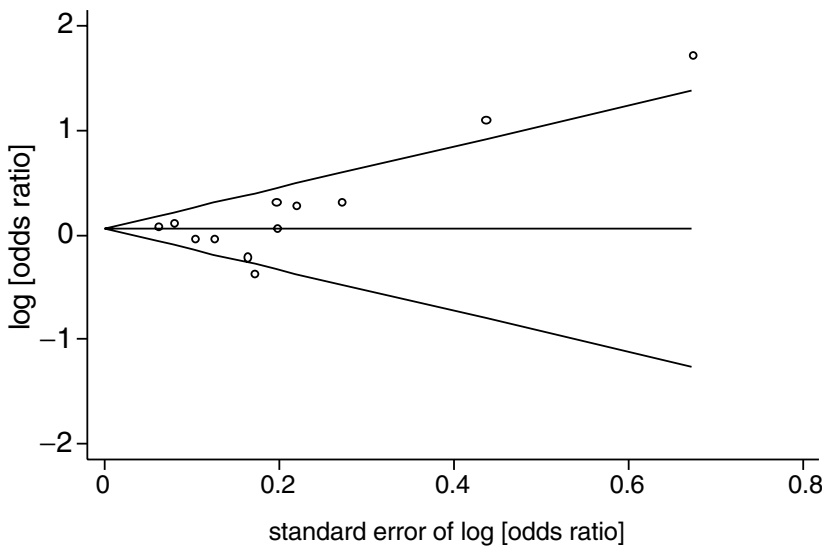

Figure 2 Funnel plot of studies of association of smoking prior to first birth and breast cancer risk.

\section{Study limitations}

The majority of our cases were prevalent and survivor bias may be an important limitation. Breast cancer in the UK is associated with a survival rate of $70 \%$ over 5 years (Coleman et al, 1999). Our study, and other case-control studies of this association, may therefore exclude a number of women with aggressive disease. If smoking prior to first birth is strongly associated with survival, then we may have missed an important association. However, two prospective studies of incident cases only did not find an association (Egan et al, 2002; Reynolds et al, 2004), and when we restricted our analyses to incident cases only, although imprecise there was no evidence of an association.

Breast cancer cases were not confirmed histologically and selfreported breast cancer may be inaccurate. However, over $80 \%$ of the cases were identified from at least two sources, including medical records, cancer registers or self-report. Medical record and cancer register cases are likely to have been confirmed by

\section{REFERENCES}

Adami HO, Lund E, Bergstrom R, Meirik O (1988) Cigarette smoking, alcohol consumption and risk of breast cancer in young women. $\mathrm{Br} J$ Cancer 58: $832-837$

Band PR, Le ND, Fang R, Deschamps M (2002) Carcinogenic and endocrine disrupting effects of cigarette smoke and risk of breast cancer. Lancet 360: $1044-1049$

Baron JA, Newcomb PA, Longnecker MP, Mittendorf R, Storer BE, Clapp RW, Bogdan G, Yuen J (1996) Cigarette smoking and breast cancer. Cancer Epidemiol Biomarkers Prev 5: $399-403$

Chu SY, Stroup NE, Wingo PA, Lee NC, Peterson HB, Gwinn ML (1990) Cigarette smoking and the risk of breast cancer. Am J Epidemiol 131: $244-253$

Coleman MP, Babb P, Damecki P, Groscalude P, Hanjos S, Jones J (1999) Cancer Survival Trends in England and Wales 1971-1995: Deprivation and NHS Region. London: Stationary Office

DerSimonian R, Laird N (1986) Meta-analysis in clinical trials. Cont Clin Trials 7: $177-188$

Egan KM, Stampfer MJ, Hunter D, Hankinson S, Rosner BA, Holmes M, Willett WC, Colditz GA, Nurses' HS (2002) Active and passive smoking in breast cancer: prospective results from the Nurses' Health Study. Epidemiology 13: 138-145

Ewertz M (1990) Smoking and breast cancer risk in Denmark. Cancer Causes Control 1: 31-37

Ewertz M (1993) Breast cancer in Denmark. Incidence, risk factors, and characteristics of survival. Acta Oncol 32: 595-615 histological reports and when sensitivity analyses were performed using breast cancer data from each of just one of the three sources (cancer register, general practice medical records, self-report) the results were unchanged. By combining information from all three sources, it is likely that most cases have been identified.

An important limitation of our, and other, studies is the small number of cases and hence imprecision of the results. This highlights the importance of pooling results from all studies in order to provide a precise estimate of the overall effect.

\section{Smoking after first birth}

Although imprecise and not an a priori hypothesis, our results suggest that smoking solely after a woman's first birth may be protective against breast cancer. Two other studies have also found reduced risk of breast cancer associated with smoking only in the period after a first birth (Band et al, 2002; Lash and Aschengrau, 2002). Some compounds in cigarettes inhibit the aromatisation of androgens to oestrogens and enhance the formation of oestrodiol metabolites with low oestrogenic activity (Michnovicz et al, 1986; Kadohama et al, 1993). Hence it has been suggested that smoking in later life may be associated with reduced risk of breast cancer through mechanisms related to decreased oestrogen activity (Band et al, 2002). However, results in this area have been inconsistent with two studies finding increased risk of breast cancer among women who begin smoking only after the birth of their first child (Lash and Aschengrau, 1999; Kropp and Chang-Claude, 2002), and two prospective studies found no association (Egan et al, 2002; Reynolds et al, 2004).

\section{CONCLUSION}

Smoking around the time of breast tissue development and before full differentiation - between puberty and prior to completion of the first pregnancy - is not associated with increased risk of breast cancer. Smoking is associated with a number of adverse health outcomes and young women should be discouraged from taking up smoking irrespective of any association or lack of association with breast cancer.
Field NA, Baptiste MS, Nasca PC, Metzger BB (1992) Cigarette smoking and breast cancer. Int J Epidemiol 21: 842-848

Fink AK, Lash TL (2003) A null association between smoking during pregnancy and breast cancer using Massachusetts registry data (United States). Cancer Causes Control 14: 497-503

Hsieh CC, Trichopoulos D, Katsouyanni K, Yuasa S (1990) Age at menarche, age at menopause, height and obesity as risk factors for breast cancer: associations and interactions in an international casecontrol study. Int J Cancer 46: 796-800

Hunter DJ, Hankinson SE, Hough H, Gertig DM, Garcia-Closas M, Spiegelman D, Manson JE, Colditz GA, Willett WC, Speizer FE, Kelsey K (1997) A prospective study of NAT2 acetylation genotype, cigarette smoking, and risk of breast cancer. Carcinogenesis 18: 2127-2132

Innes KE, Byers TE (2001) Smoking during pregnancy and breast cancer risk in very young women (United States). Cancer Causes Control 12: $179-185$

Kadohama N, Shintani K, Osawa Y (1993) Tobacco alkaloid derivatives as inhibitors of breast cancer aromatase. Cancer Lett 75: 175-182

Kropp S, Chang-Claude J (2002) Active and passive smoking and risk of breast cancer by age 50 years among German women. Am J Epidemiol 156: $616-626$

Lash TL, Aschengrau A (1999) Active and passive cigarette smoking and the occurrence of breast cancer. Am J Epidemiol 149: 5-12

Lash TL, Aschengrau A (2002) A null association between active or passive cigarette smoking and breast cancer risk. Breast Cancer Res Treat 75: $181-184$ 
Lawlor DA, Bedford C, Taylor M, Ebrahim S (2003a) Geographical variation in cardiovascular disease, risk factors, and their control in older women: British Women's Heart and Health Study. J Epidemiol Community Health 57: $134-140$

Lawlor DA, Okasha M, Gunnell D, Davey Smith G, Ebrahim S (2003b) Associations of adult measures of childhood growth with breast cancer: findings from the British Women's Heart and Health Study. Br J Cancer 89: $81-87$

MacMahon B, Cole P, Lin TM, Lowe CR, Mirra AP, Ravnihar B, Salber EJ, Valaoras VG, Yuasa S (1970) Age at first birth and breast cancer risk. Bull World Health Organization 43: 209-221

Michnovicz JJ, Hershcopf RJ, Naganuma H, Bradlow HL, Fishman J (1986) Increased 2-hydroxylation of estradiol as a possible mechanism for the anti-estrogenic effect of cigarette smoking. $N$ Engl J Med 315: $1305-1309$

O’Connell DL, Hulka BS, Chambless LE, Wilkinson WE, Deubner DC (1987) Cigarette smoking, alcohol consumption, and breast cancer risk. $J$ Natl Cancer Inst 78: 229-234
Office of Population Censuses and Surveys (1980) Classification of Occupations and Coding Index. London: HM Stationary Office

Reynolds P, Hurley S, Goldberg DE, Anton-Culver H, Bernstein L, Deapen D, Horn-Ross PL, Peel D, Pinder R, Ross RK, West D, Wright WE, Ziogas A (2004) Active smoking, household passive smoking, and breast cancer: evidence from the California Teachers Study. J Natl Cancer Inst 96: 29 37

Rohan TE, Baron JA (1989) Cigarette smoking and breast cancer. Am J Epidemiol 129: $36-42$

Smith SJ, Deacon JM, Chilvers CE (1994) Alcohol, smoking, passive smoking and caffeine in relation to breast cancer risk in young women. UK National Case-Control Study Group. Br J Cancer 70: 112-119

Sterne JAC, Bradburn MJ, Egger M (2001) Meta-analysis in Stata ${ }^{\mathrm{TM}}$. In Systematic Reviews in Health Care. Meta-Analysis in Context, Egger M, Davey Smith G, Altman DG (eds) pp 347-369, London: BMAbooks

Terry PD, Rohan TE (2002) Cigarette smoking and the risk of breast cancer in women: a review of the literature. Cancer Epidemiol Biomarkers Prev 11: $953-971$ 